\title{
Overcoming the fundamental light-extraction efficiency limitations of deep ultraviolet light-emitting diodes by utilizing transverse-magnetic-dominant emission
}

\author{
Dong Yeong Kim ${ }^{1, *}$, Jun Hyuk Park ${ }^{1, *}$, Jong Won Lee ${ }^{1}$, Sunyong Hwang ${ }^{1}$, Seung Jae Oh ${ }^{1}$, Jungsub Kim², \\ Cheolsoo Sone ${ }^{2}$, E. Fred Schubert ${ }^{3}$ and Jong Kyu Kim ${ }^{1}$
}

While the demand for deep ultraviolet (DUV) light sources is rapidly growing, the efficiency of current AIGaN-based DUV light-emitting diodes (LEDs) remains very low due to their fundamentally limited light-extraction efficiency (LEE), calling for a novel LEE-enhancing approach to deliver a real breakthrough. Here, we propose sidewall emission-enhanced (SEE) DUV LEDs having multiple light-emitting mesa stripes to utilize inherently strong transverse-magnetic polarized light from the AIGaN active region and three-dimensional reflectors between the stripes. The SEE DUV LEDs show much enhanced light output power with a strongly upward-directed emission due to the exposed sidewall of the active region and Al-coated selective-area-grown n-type GaN micro-reflectors. The devices also show reduced operating voltage due to better $\mathrm{n}$-type ohmic contact formed on the regrown $\mathrm{n}$-GaN stripes when compared with conventional LEDs. Accordingly, the proposed approach simultaneously improves optical and electrical properties. In addition, strategies to further enhance the LEE up to the theoretical optimum value and control emission directionality are discussed.

Light: Science \& Applications (2015) 4, e263; doi:10.1038/Isa.2015.36; published online 10 April 2015

Keywords: deep ultraviolet; light-emitting diodes; light-extraction efficiency

\section{INTRODUCTION}

The electromagnetic wavelength range between $200 \mathrm{~nm}$ and $280 \mathrm{~nm}$ is referred to as ultraviolet (UV)-C or, simply, as deep-ultraviolet (DUV). One of the most remarkable characteristics of DUV light is that it can strongly damage bacteria and viruses, and for this reason, it has been used in air/water/food purification and sterilization to protect humans from serious health threats. ${ }^{1-3}$ Other major applications of DUV light include polymer curing, semiconductor photolithography, and high-density optical recording. ${ }^{3-6}$ Currently, man-made DUV light sources are employed for those applications because natural DUV light from the Sun is absorbed by ozone and $\mathrm{O}_{2}$ molecules in the Earth's stratosphere. ${ }^{7}$ Hg-vapor lamps have been widely used as conventional DUV light sources for over 100 years despite their inefficient energy usage, bulky and heavy structure, and serious environmental burden arising from mercury (Hg) usage and disposal. New UV light sources based on AlGaN-based UV light-emitting diodes (LEDs) have attracted much attention due to their many advantages over $\mathrm{Hg}$-vapor lamps including compact size, low power consumption, instant startup, environmental friendliness and wavelength tunability by adjusting the $\mathrm{Al}$ content in the $\mathrm{Al}_{x} \mathrm{Ga}_{1-x} \mathrm{~N}$ active region $(210 \mathrm{~nm}$ for AlN $\sim 365 \mathrm{~nm}$ for GaN). ${ }^{8,9}$ Recent vibrant research on AlGaN-based UV LED has led to remarkable developments in external-quantum efficiency (EQE). However, the EQE decreases rapidly as the wavelength becomes shorter (by increasing the $\mathrm{Al}$ content in the $\mathrm{Al}_{x} \mathrm{Ga}_{1-} \mathrm{N}$ ). Although Shatalov et al. ${ }^{10}$ reported AlGaN DUV LEDs with an EQE above $10 \%$ at $278 \mathrm{~nm}$, typical EQE values of commercial DUV LEDs are less than $5 \%$. $^{1}$

The dominant reason for such a low EQE is the poor light-extraction efficiency (LEE) caused by the strongly anisotropic light emission that is mostly in a direction within the $c$-plane of $\mathrm{Al}_{x} \mathrm{Ga}_{1-x} \mathrm{~N}^{1,8,9}$ The strongly anisotropic emission, called sidewall emission in this study, is due to the unique valence band (VB) structure of AlGaN at high $\mathrm{Al}$ contents $(x>\sim 0.25) .{ }^{11-13}$ The topmost VB of high-Al-content AlGaN is the crystal field split-off hole $(\mathrm{CH})$ band, which has a $\mathrm{P}_{Z}$-orbital-like property. Therefore, AlGaN dominantly emits transverse magnetic (TM)-polarized light propagating perpendicular to the $c$-axis with the light's electric field parallel to the $c$-axis $(E / / c)$. However, conventional LED chip geometries favor extracting light propagating parallel to the $c$-axis, and thus, the LEEs of AlGaN-based DUV LEDs with conventional chip geometries do not exceed $10 \% .^{10,14,15}$ Conventional LEE-enhancing techniques-such as surface roughening, ${ }^{16,17}$ substrate patterning ${ }^{18}$ and incorporating anti-reflective coatings, ${ }^{19}$ photonic crystals ${ }^{20}$ and surface plasmonics ${ }^{21}$ — are effective for GaInN-based visible LEDs but have a negligible effect on AlGaN DUV LEDs. ${ }^{14}$

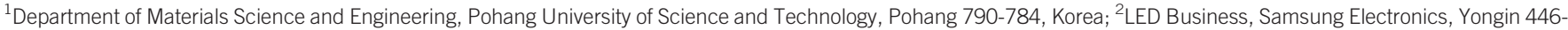
920, Korea and ${ }^{3}$ Future Chips Constellation, Department of Electrical, Computer, and Systems Engineering, Rensselaer Polytechnic Institute, Troy, NY 12180, USA

*These authors contributed equally to this work.

Correspondence: JK Kim, Department of Materials Science and Engineering, Pohang University of Science and Technology, Pohang 790-784, Korea

E-mail: kimjk@postech.ac.kr

Received 22 October 2014; revised 31 December 2014; accepted 3 January 2015; accepted article preview online 6 January 2015 
According to recent finite difference time domain calculations, the extraction of TM-polarized DUV light is $>10$ times lower than that of transverse electric (TE)-polarized light. ${ }^{22}$ Thus, a new strategy that is totally different from conventional strategies, utilizing the strong sidewall emission in AlGaN DUV LEDs, should be developed to demonstrate DUV LEDs with high LEEs, and thus, high EQEs.

Here, we present a new LEE-enhancing concept for AlGaN DUV LEDs. The proposed DUV LEDs, called sidewall emission-enhanced (SEE) DUV LEDs, have multiple narrow mesa stripe structures exposing the sidewall of the multiple-quantum well (MQW) active region to efficiently extract TM-polarized DUV light. In addition, Al-coated selective-area-grown n-type GaN micro-mirrors are located between the exposed MQW sidewalls to reflect the DUV photons in the upward direction. The fundamental light emission characteristics of AlGaNbased DUV LEDs were considered theoretically, and the effectiveness of the proposed LEE-enhancing strategy was experimentally verified with an $\mathrm{Al}_{0.4} \mathrm{Ga}_{0.6} \mathrm{~N}$-based SEE DUV LED ( $\left.\lambda_{\text {peak }}=285 \mathrm{~nm}\right)$. The SEE DUV LEDs show not only strong improvement in light extraction through the sidewalls of the MQWs and enhanced directional emission toward the top enabled by the Al micro-mirrors, but also a much lower operating voltage compared with conventional DUV LEDs.

\section{MATERIALS AND METHODS}

\section{Growth of the AlGaN-based DUV LED structure}

A conventional $\mathrm{Al}_{0.4} \mathrm{Ga}_{0.6} \mathrm{~N} / \mathrm{Al}_{0.55} \mathrm{Ga}_{0.45} \mathrm{~N}$ MQW DUV LED wafer (for $\lambda_{\text {peak }}=285 \mathrm{~nm}$ DUV emission) was grown on a 4 -inch $c$-plane sapphire substrate by metal-organic vapor phase epitaxy. The 285-nm DUV LED consists of an AlN buffer layer, AlGaN/AlN superlattice buffer layers, a $2 \mu \mathrm{m}$-thick Si-doped ( $\mathrm{Si}$ concentration $[\mathrm{Si}]=1 \times 10^{19} \mathrm{~cm}^{-3}$ ) $\mathrm{Al}_{0.55} \mathrm{Ga}_{0.45} \mathrm{~N}$ n-type cladding layer, five-period MQWs composed of $3 \mathrm{~nm}$-thick undoped $\mathrm{Al}_{0.4} \mathrm{Ga}_{0.6} \mathrm{~N}$ wells and $10 \mathrm{~nm}$-thick undoped $\mathrm{Al}_{0.55} \mathrm{Ga}_{0.45} \mathrm{~N}$ barriers, a $20 \mathrm{~nm}$-thick $\mathrm{Mg}$-doped ( $\mathrm{Mg}$ concentration $\left.[\mathrm{Mg}]=2 \times 10^{20} \mathrm{~cm}^{-3}\right) \mathrm{Al}_{0.7} \mathrm{Ga}_{0.3} \mathrm{~N}$ electron blocking layer, a $\mathrm{Mg}$-doped (Mg concentration $[\mathrm{Mg}]=2 \times 10^{20} \mathrm{~cm}^{-3}$ ) AlGaN p-type cladding layer having $\mathrm{Al}$ content grading from $65 \%$ to $0 \%$, and a $20 \mathrm{~nm}$-thick $\mathrm{Mg}$ doped (Mg concentration $[\mathrm{Mg}]>2 \times 10^{20} \mathrm{~cm}^{-3}$ ) GaN p-type contact formation layer. The energy band diagram of the DUV LED structure is shown in Supplementary Fig. S1.

\section{SEE DUV LEDs}

Figure $1 \mathrm{a}$ and $1 \mathrm{~b}$ schematically shows the structures of the reference and the SEE DUV LED, respectively. Both LEDs have multiple narrow mesa stripe structures to expose the sidewall of the MQWs for better extraction of the sidewall-emitting TM-polarized DUV light. While ohmic contacts on exposed n-type AlGaN are located between the active mesa stripes for the reference LED, Al-coated selective-areagrown n-type GaN micro-mirrors are located between the mesas for the SEE DUV LED to redirect DUV photons emitted from the sidewall toward the top. The effect of the multiple narrow mesa stripe structures on light extraction and the effect of the micro-mirrors on the emission pattern are revealed by FEM simulations using COMSOL multiphysics (Supplementary Fig. S2)

\section{Fabrication of SEE DUV LEDs}

AlGaN SEE DUV LEDs with different numbers of stripes (from 5 to 45; Table 1) formed on the $1 \times 1 \mathrm{~mm}^{2}$ chip area were fabricated to investigate the effect of the exposed sidewall area of the active region on light extraction. The detailed fabrication process of the SEE DUV LEDs is described in the Supplementary Fig. S3. Here, we briefly introduce how to fabricate SEE DUV LEDs. The overall fabrication follows a conventional LED fabrication method. First, narrow mesa stripes are formed by photolithographic patterning followed by reactive ion etching with $\mathrm{BCl}_{3}$ and $\mathrm{Cl}_{2}$ gases (target etching depth is $1 \mu \mathrm{m}$ ). Then, $50 \mathrm{~nm}$ thick $\mathrm{SiO}_{2}$ is deposited by plasma-enhanced chemical vapor deposition and selectively opened by photolithographic patterning and wet etching with buffered oxide etchant. The $\mathrm{n}-\mathrm{GaN}$ microstructures are grown on the opening on the n-AlGaN surface by selective area growth ${ }^{23,24}$ by metal-organic vapor phase epitaxy regrowth for $3 \mathrm{~min}$ at $1000{ }^{\circ} \mathrm{C}$ at $200 \mathrm{mbar}$. The V/III ratio is 606 , and the silane flow is $50 \mathrm{sccm}$. As a result of the selective area growth, three dimensional n-type GaN microstructures with isosceles trapezoidal stripes (from stripe-shaped openings) are obtained. ${ }^{25}$ Then, the $n$-contact (Ti/Al/Ni/Au) is deposited on the regrown $\mathrm{n}-\mathrm{GaN}$ microstructures as well as the exposed nAlGaN surface by electron-beam/thermal evaporation and annealed at $900{ }^{\circ} \mathrm{C}$ for $2 \mathrm{~min}$ in ambient $\mathrm{N}_{2}$. The p-contact (Ni/Au) is deposited and annealed at $650{ }^{\circ} \mathrm{C}$ for $1 \mathrm{~min}$ in ambient air. Then, aluminum $(150 \mathrm{~nm})$ is thermally deposited on the grown $\mathrm{n}-\mathrm{GaN}$ stripes. The final step is $\mathrm{Ti} /$ $\mathrm{Au}$ contact pad metallization for the $\mathrm{n}$ - and p-type contacts.

\section{RESULTS AND DISCUSSION}

\section{Inherently strong sidewall emission in AlGaN-based DUV LEDs} Fundamentally, light generated by the radiative recombination of conduction band (CB) electrons with VB holes is described by Fermi's golden rule, where the polarization of the generated light is
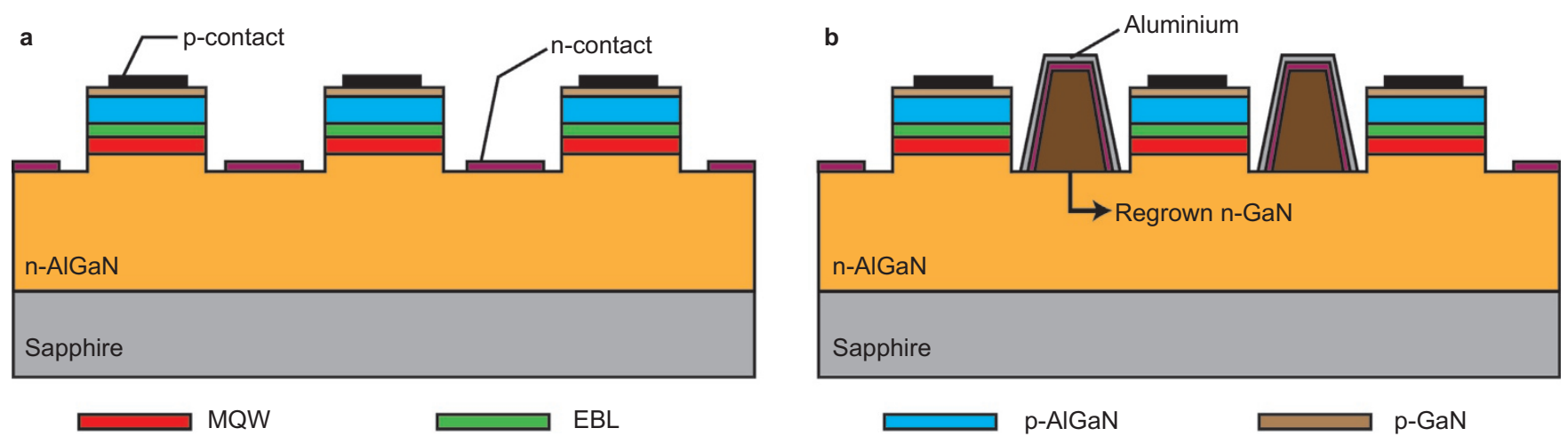

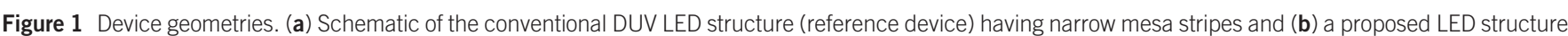

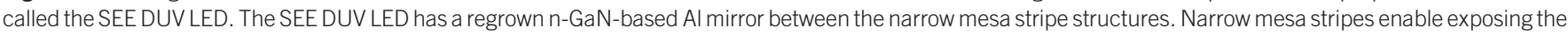
sidewall of the active region and effectively extracting sidewall emitting DUV photons. AI mirrors redirect the extracted photons from the sidewall toward the top. DUV, deep ultraviolet; LED, light-emitting diode; SEE, sidewall emission-enhanced. 
Table 1 Various SEE DUV LEDs having a $1 \times 1 \mathrm{~mm}^{2}$ device area. As the number of stripes increases, the perimeter of the active mesa and the $n$-contact area increase and the area of the active mesa decreases

\begin{tabular}{lccc}
$\begin{array}{l}\text { Number of } \\
\text { stripes }\end{array}$ & $\begin{array}{c}\text { Perimeter of active } \\
\text { mesa }(\mathrm{mm})\end{array}$ & $\begin{array}{c}\text { Area of active mesa } \\
\left(\times 10^{4} \mu \mathrm{m}^{2}\right)\end{array}$ & $\begin{array}{c}\text { n-contact area } \\
\left(\times 10^{4} \mu \mathrm{m}^{2}\right)\end{array}$ \\
\hline 5 & 10.81 & 83.86 & 12.14 \\
10 & 19.67 & 79.02 & 14.33 \\
15 & 28.34 & 73.08 & 17.67 \\
20 & 37.34 & 67.53 & 20.51 \\
25 & 46.01 & 61.72 & 23.73 \\
30 & 55.01 & 56.05 & 26.70 \\
35 & 63.68 & 50.28 & 29.86 \\
40 & 72.51 & 46.65 & 32.83 \\
45 & 81.35 & 38.26 & 36.02 \\
\hline
\end{tabular}

DUV, deep ultraviolet; LED, light-emitting diode; SEE, sidewall emission-enhanced.

determined by the $\mathrm{CB}$ states and the $\mathrm{VB}$ states involved in the transition. Coldren and Corzine ${ }^{26}$ showed that the total spontaneous emission rate $R_{\mathrm{sp}}$ is given by

$$
R_{\mathrm{sp}}=C \times \int d E_{21} h v_{21}\left|\bar{M}_{\mathrm{T}}\left(E_{21}\right)\right|^{2} \rho_{\mathrm{r}}\left(E_{21}\right) f_{2}\left(1-f_{1}\right)
$$

Here, $C$ is a constant that includes the active-region area, thickness, and the carrier density, $E_{21}$ is the transition energy, $h$ is the Planck constant, $v_{21}$ is the frequency of the generated light, $\rho_{\mathrm{r}}$ is the reduced density of states, $f_{1}$ and $f_{2}$ are the Fermi-Dirac distributions for electrons and holes, and $\left|M_{T}\right|^{2}$ is the transition matrix element given by

$$
\left|\bar{M}_{\mathrm{T}}\left(E_{21}\right)\right|^{2}=\frac{1}{3}\left|\left\langle F_{2} \mid F_{1}\right\rangle\right|^{2} \sum_{\hat{e}_{x}, \hat{e}_{y}, \hat{e}_{z}}\left|\left\langle u_{\mathrm{c}}|\hat{\boldsymbol{e}} \cdot \boldsymbol{p}| u_{\mathrm{v}}\right\rangle\right|^{2}
$$

where $F_{1}$ and $F_{2}$ are the envelope functions of carriers and $u_{c}$ and $u_{\mathrm{v}}$ are the Bloch functions of the CBs and VBs, respectively. The term $\left\langle u_{\mathrm{c}}|\hat{\boldsymbol{e}} \cdot \boldsymbol{p}| u_{\mathrm{v}}\right\rangle$ represents the polarization selection rule determining the allowed light polarization direction of the emitted light. Momentum transfer between $\mathrm{CBs}$ and VBs along the three principle directional unit vectors, $\hat{\boldsymbol{e}}_{x}, \hat{\boldsymbol{e}}_{y}, \hat{\boldsymbol{e}}_{z}$ determines the electric field direction (polarization direction). Thus, the symmetry of the $\mathrm{CB}$ and $\mathrm{VB}$ is the polarization-determining factor.

The Bloch function for states near the CB-edge at $\boldsymbol{k}=0$ has same symmetry characteristics as a $|S\rangle$. Meanwhile, all VBs including the heavy hole $(\mathrm{HH})$ band, the light hole $(\mathrm{LH})$ band, and the $\mathrm{CH}$ band at those maxima have the symmetry of P-orbitals. Both $\mathrm{HH}$ and $\mathrm{LH}$ possess two P-like orbital functions, $|X\rangle$ and $|Y\rangle$ in the $c$-plane along the $a$ - and $b$-axis, and the $\mathrm{CH}$ band has $|Z\rangle$-like states. Therefore, the light polarization direction is determined by the symmetry of the topmost VB. The crystal field split-off energy $\left(\Delta_{\mathrm{CR}}\right)$ determines the topmost state among the three VBs. ${ }^{27}$ When $\Delta_{\mathrm{CR}}$ is positive, the topmost $\mathrm{VB}$ is the $\mathrm{HH}$ band, and it is converted to the $\mathrm{CH}$ band when $\Delta_{\mathrm{CR}}$ becomes negative. Figure 2 shows the dispersion relations of the VBs for $c$-plane $\mathrm{GaN}, \mathrm{Al}_{0.4} \mathrm{Ga}_{0.6} \mathrm{~N}$, and $\mathrm{AlN}$ calculated by a $6 \times 6$ effectivemass Hamiltonian matrix for the wurtzite structure based on the $\mathrm{k} \cdot \mathrm{p}$ perturbation theory as derived by Chuang and Chang. ${ }^{28}$ Due to the positive $\Delta_{\mathrm{CR}}$, the topmost $\mathrm{VB}$ of $\mathrm{GaN}$ is the $\mathrm{HH}$ band (Figure 2a), whose basis function is a linear combination of the $\mathrm{P}_{X^{-}}$and $\mathrm{P}_{Y^{-}}$orbital wave functions. In contrast, because of the negative $\Delta_{\mathrm{CR}}$ the topmost $\mathrm{VB}$ of $\mathrm{AlN}$ is the $\mathrm{CH}$ band, whose basis function strongly reflects the $\mathrm{P}_{Z}$-orbital wave function, as shown in Figure $2 \mathrm{c}$. By considering the momentum transfer between $\mathrm{CB}$ and $\mathrm{VB}$ based on their wave function symmetry, GaN and AlN predominantly generate in-plane (TE) polarized light and out-of-plane (TM) polarized light, respectively, while AlGaN has an intermediate property. For bulk $\mathrm{Al}_{x} \mathrm{Ga}_{1-{ }_{x} \mathrm{~N}}$, the sign of $\Delta_{\mathrm{CR}}$ changes from positive to negative when $x$ becomes greater than $0.25{ }^{13}$ For the AlGaN MQW DUV LEDs, this transition occurs at an $\mathrm{Al}$ content higher than 0.25 due to the quantum confinement effect and the strain in the QWs. For AlGaN DUV LEDs grown on sapphire, the transition generally occurs at the wavelength of approximately $295 \mathrm{~nm}$ corresponding to an $\mathrm{Al}$ content of approximately $35 \% .^{29}$ Thus, high Al-content AlGaN more closely resembles AlN than $\mathrm{GaN}$, and thus, generates predominantly TM-polarized light. The calculated $\mathrm{VB}$ structure of $\mathrm{Al}_{0.4} \mathrm{Ga}_{0.6} \mathrm{~N}$, used as an active layer for 285-nm DUV LED is shown in Figure 2b. The topmost $\mathrm{VB}$ of $\mathrm{Al}_{0.4} \mathrm{Ga}_{0.6} \mathrm{~N}$ is the $\mathrm{CH}$ band, so the 285-nm DUV LED fabricated in this study is expected to generate predominantly TM-polarized light. The polarization ratio, $\left(I_{\mathrm{TE}}-I_{\mathrm{TM}}\right) /\left(I_{\mathrm{TE}}+I_{\mathrm{TM}}\right)$, of the 285-nm DUV LED is measured to be 0.067, indicating a strong TM-polarization (Supplementary Fig. S4), consistent with the expectation and previous report. ${ }^{29}$

Figure 3 shows a time-averaged power flow radiated from dipole sources. Figure $3 \mathrm{a}$ is the radiation pattern of a single electric dipole surrounded by air. Its dipole moment is along the $y$-axis. The figure clearly shows that the anisotropic emission with light propagation perpendicular to the direction of dipole moment, i.e., the $x$-axis in this calculation, is much stronger than the emission along directions parallel to dipole moment. When there are two incoherent dipole

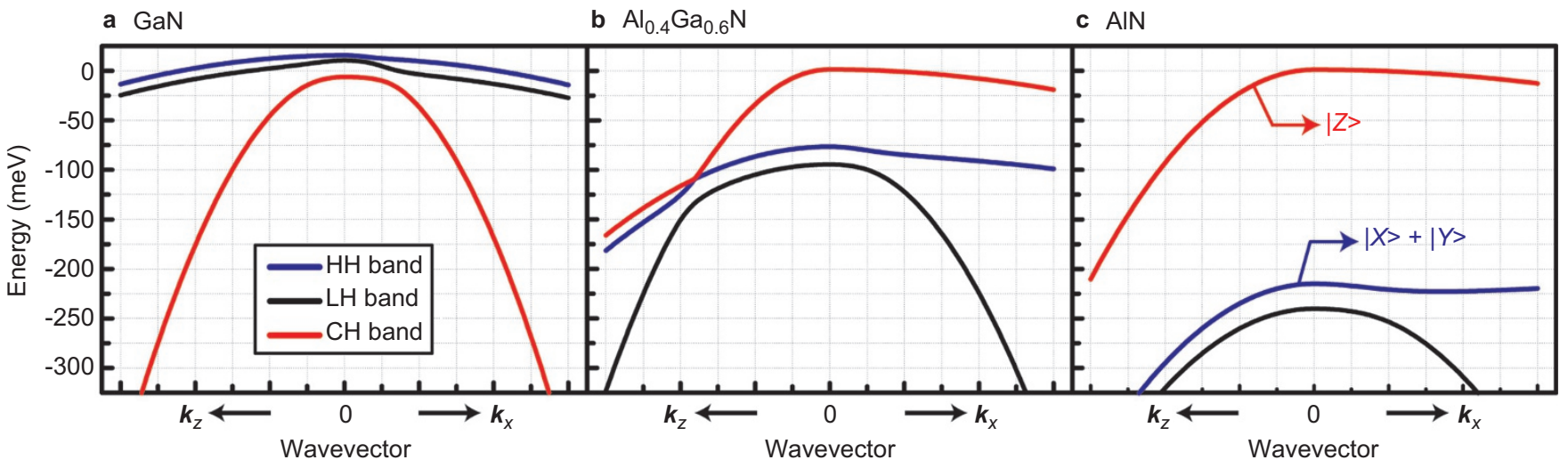

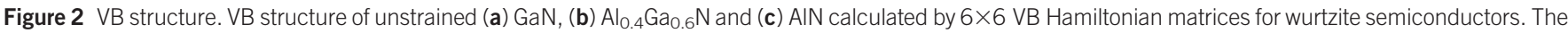

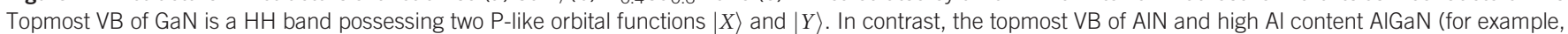
$\mathrm{Al}_{0.4} \mathrm{Ga}_{0.6} \mathrm{~N}$ ) is a $\mathrm{CH}$ band possessing $|Z\rangle$-like states. $\mathrm{CH}$, crystal field split-off hole; $\mathrm{HB}$, heavy hole; VB, valence band. 

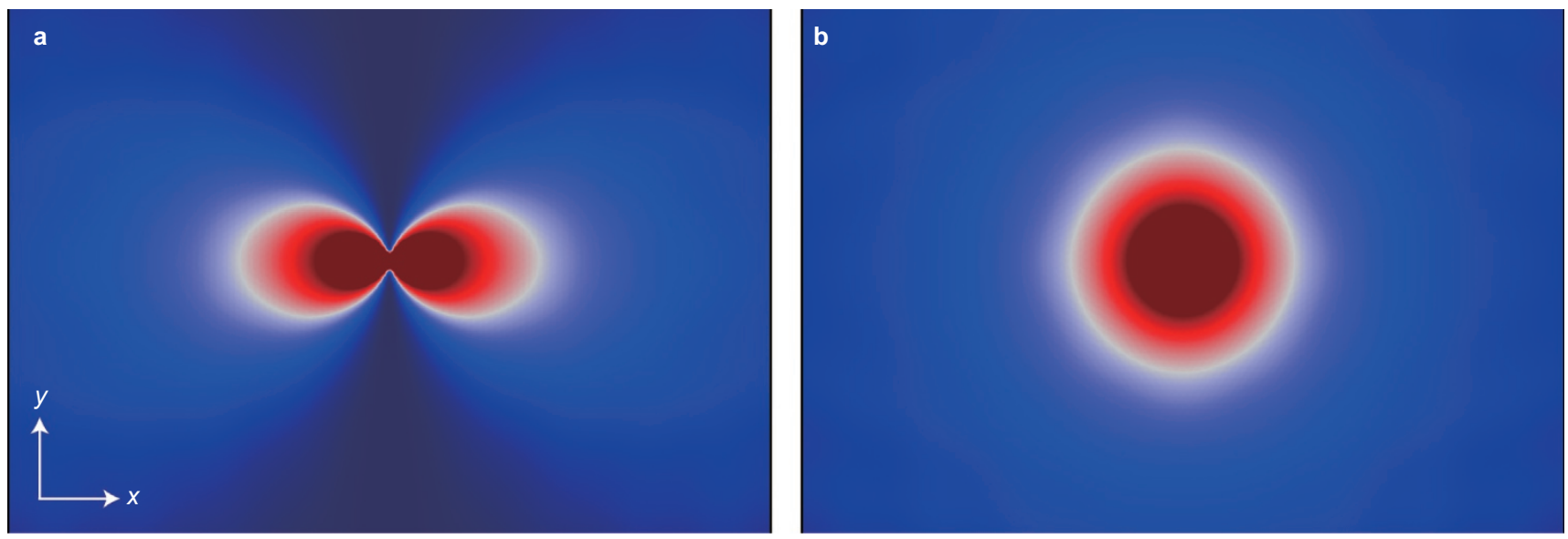

Figure 3 Dipole emission patterns. Emission pattern, simulated by COMSOL multiphysics, of (a) a single electric dipole source whose dipole moment is along the $y$-axis and (b) two incoherent dipole sources whose dipole moment directions are orthogonal, i.e., one electric dipole is along the $x$-axis, and another dipole is along the $y$-axis. In the case of (a), a single electric dipole generates anisotropic light perpendicular to its dipole moment direction, but in the case of (b), two incoherent dipoles generate isotropic light.

sources whose dipole moment directions are orthogonal, i.e., one electric dipole is along the $x$-axis, and another dipole is along the $y$ axis, isotropic light emission is generated, as shown in Figure $3 \mathrm{~b}$. Based on the dipole radiation approximation ${ }^{30}$ described by

$$
\int u_{\mathrm{c}}^{*}(\hat{\boldsymbol{e}} \cdot \boldsymbol{p}) u_{\mathrm{v}} \cdot \mathrm{e}^{-i \cdot \vec{k} \cdot \vec{r}} \mathrm{~d} \tau \sim \int u_{\mathrm{c}}^{*}(\hat{\boldsymbol{e}} \cdot \boldsymbol{p}) u_{\mathrm{v}} \mathrm{d} \tau=m \frac{\mathrm{d}}{\mathrm{d} t} \int u_{\mathrm{c}}^{*}(\hat{\boldsymbol{e}} \cdot \boldsymbol{r}) u_{\mathrm{v}} \mathrm{d} \tau
$$

the emission characteristics of $\mathrm{GaN}$ and $\mathrm{AlN}$ can be predicted. For $\mathrm{GaN}$, the $\mathrm{HH}$-band recombination is approximated by two incoherent dipole sources aligned along the $x$ - and $y$-directions, consistent with the symmetry of the HH band. Therefore, predominantly isotropic TE-polarized light is generated. However, the emission from AlN can be regarded as an outcome of a single dipole source aligned along the $z$-direction due to the $\mathrm{P}_{Z}$-orbital-like symmetry of the $\mathrm{CH}$ band. Thus, $\mathrm{AlN}$ emission is strongly anisotropic and TM-polarized so that the emission in the $c$-plane is much stronger than that perpendicular to the $c$-plane.

\section{SEE DUV LEDs: device geometries}

Figure 4a shows a scanning electron microscopy (SEM) image of stripe-patterned SEE DUV LEDs with 45 mesa stripes (brighter stripes in the SEM image). Regrown n-GaN stripes covered by an ohmic contact and an $\mathrm{Al}$ reflector exist between mesa stripes (darker stripes in the SEM image). Other SEE DUV LEDs having a different number of stripes are shown in Supplementary Information S5. Figure $4 \mathrm{~b}$ is the cross-sectional SEM image of the typical regrown $n-G a N$ stripes, and it has a trapezoidal shape, i.e., a wide-bottom-and-narrow-top geometry, to reflect DUV photons emitted from the mesa sidewalls upward. The inclined facet corresponds to the $\{1 \overline{1} 01\}$ facet, which is generally obtained when the window mask is along $\langle 11 \overline{2} 0\rangle$ on the $c$ plane. $^{25}$ The perimeter of the active stripe-patterned mesa structures, active area, and n-contact area of SEE DUV LEDs having different numbers of stripes are summarized in Table 1.

\section{SEE DUV LEDs: device performance}

Figure 5a shows the relative light output power (LOP) of the reference and SEE DUV LEDs at an injection current of $100 \mathrm{~mA}$ with various numbers of mesa stripes measured in the surface normal direction (on the $c$-axis) using a Si photodiode. Note that the LOP and currentvoltage characteristic measurements were performed under pulse mode to exclude a thermal effect enabled by the geometry of the SEE DUV
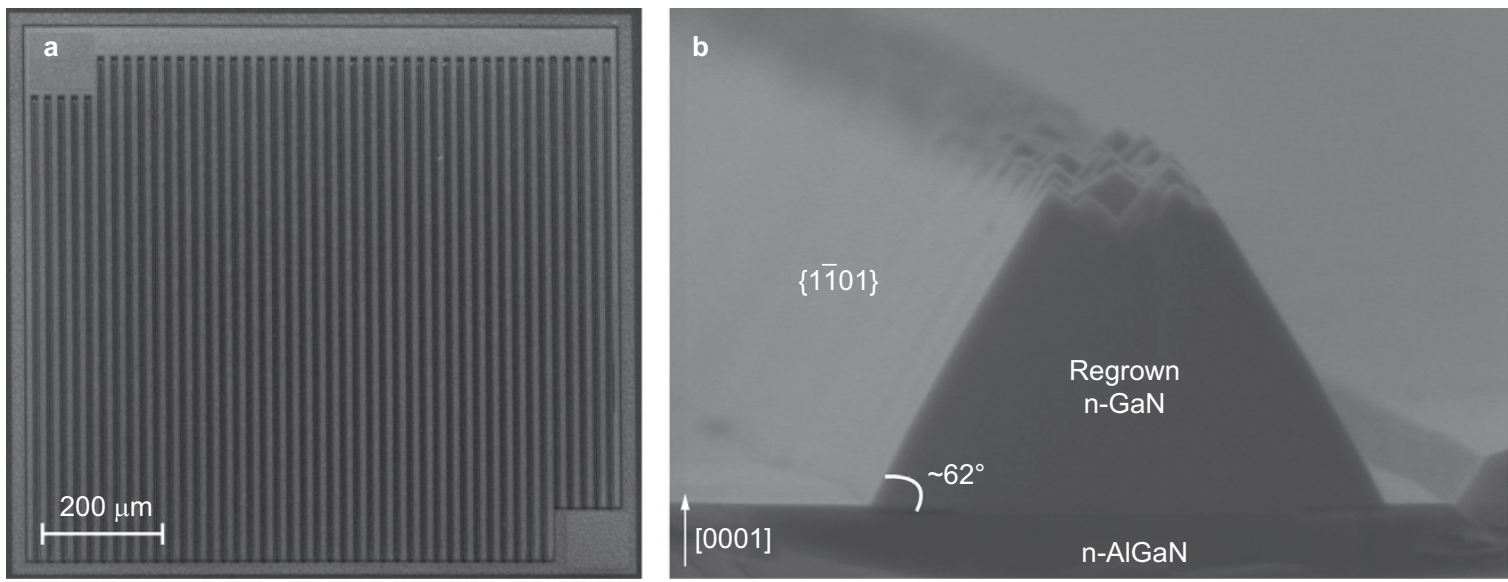

Figure 4 SEM images. SEM image of (a) an SEE DUV LED having 45 stripes. Brighter stripes and darker stripes are the mesa stripes and regrown n-GaN stripes covered by n-metal and Al, respectively. (b) Cross-sectional SEM image of typical regrown n-GaN stripes. The inclined facet corresponds to the $\{1 \overline{1} 01\}$ facet with an inclined angle of $62^{\circ}$. DUV, deep ultraviolet; LED, light-emitting diode; SEM, scanning electron microscopy. 

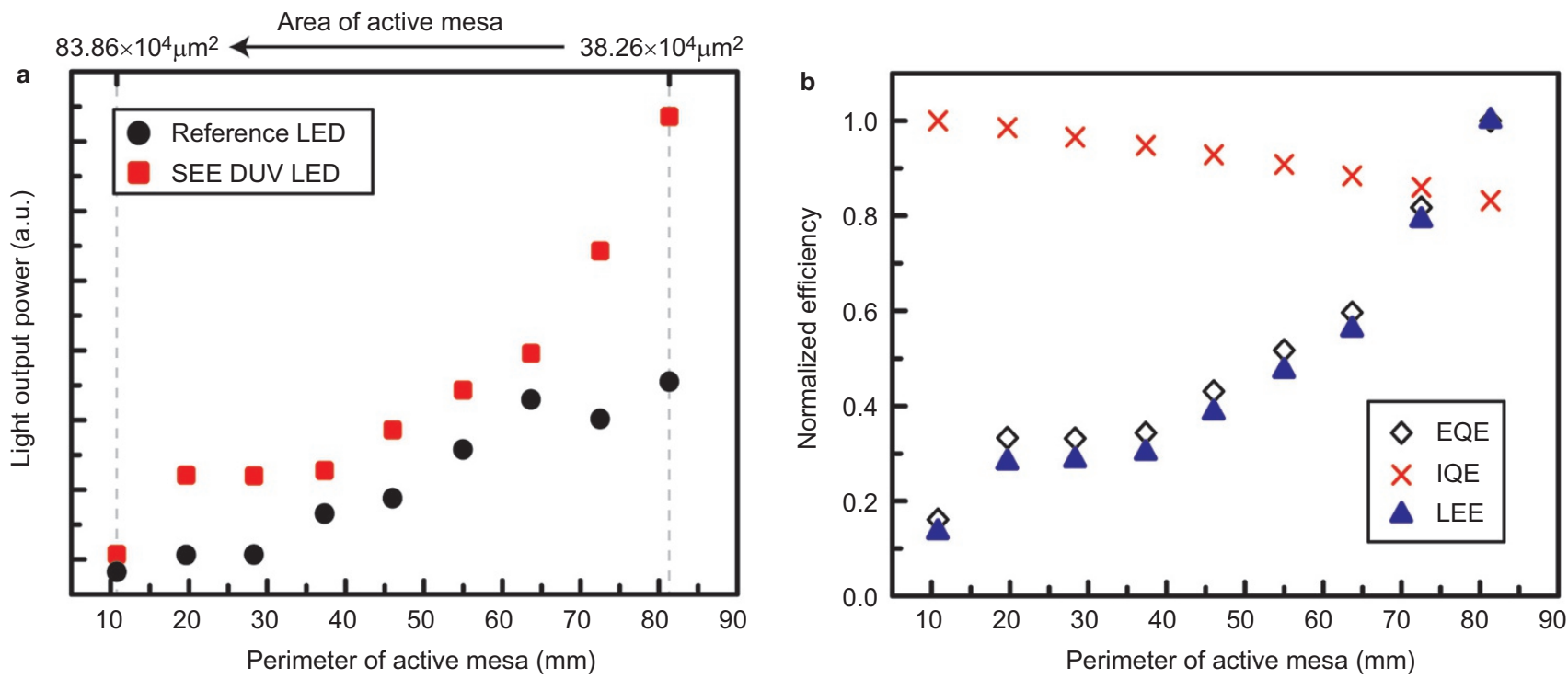

Figure 5 LOP and LEE. LOPs of the reference LEDs and SEE DUV LEDs measured in the surface normal direction at an injection current of $100 \mathrm{~mA}$ as a function of (a) the perimeter of the active region and the active area. (b) Normalized EQE, IQE and LEE of the various SEE DUV LEDs. DUV, deep ultraviolet; EQE, external-quantum efficiency; IQE, internal-quantum efficiency; LED, light-emitting diode; LEE, light-extraction efficiency; LOP, light output power; SEE, sidewall emission-enhanced.

LEDs that can effectively alleviate lateral current crowding. ${ }^{8,31-33}$ (see LOP as a function of injection current in Supplementary Fig. S6). The exposed sidewall area of the MQW active region can be quantified by the perimeter of the mesa stripes because that area is the perimeter of the active mesa stripe multiplied by the total thickness of the QWs, which is constant for all samples. The LOP increases upon increasing the perimeter of the active mesa for both the reference and SEE DUV LEDs, which is attributed to two reasons. First, DUV photons are effectively extracted through the exposed sidewall of the MQW. Secondly, the probability of re-absorption at the active region and the p-GaN contact formation layer becomes smaller by increasing the number of stripes, i.e., by increasing the perimeter of active stripes, because the travel length of DUV photons prior to being extracted becomes shorter. Compared with reference LEDs with the same number of stripes, SEE DUV LEDs show higher LOPs because a part of the emitted DUV photons through the sidewalls is more likely to be reabsorbed by the MQW and the p-GaN contact formation layer of the neighboring mesa stripes in the reference LEDs than be reflected by the $\mathrm{Al}$ reflectors in the SEE DUV LEDs. The reflection of extracted DUV photons at the $\mathrm{Al}$ mirrors increasing the LOP in the surface normal direction compared with the reference LED without $\mathrm{Al}$ mirrors will be further discussed later with an angle-dependent LOP result.

Figure $5 \mathrm{~b}$ shows the normalized EQE, internal-quantum efficiency (IQE) and LEE of SEE DUV LEDs as a function of the perimeter of the active region (Supplementary Fig. S7 for details of the calculation). As the perimeter of the active mesa increases, the IQE decreases due to the efficiency droop-i.e., the decrease in IQE with increasing injection current density. SEE DUV LEDs with a longer perimeter of the active mesa have a smaller active area and a higher current density at the same injection current and thus suffer more from IQE droop. Despite the slight decrease in IQE, the EQE increases upon increasing the perimeter due to the remarkable enhancement in LEE.

Figure $5 \mathrm{a}$ also shows the relative LOPs of the reference and SEE DUV LEDs as functions of the active mesa area. Interestingly, the LOPs of both DUV LEDs decreases upon increasing the area of the active mesa, which can be explained as follows: The area of the active region is inversely proportional to the perimeter for the same device size $\left(1 \times 1 \mathrm{~mm}^{2}\right.$, Table 1$)$, demonstrating that enhancing the extraction of DUV photons by increasing the perimeter of the sidewall of the MQW mesas is much more effective than increasing the active area where DUV photons are generated. In addition, the effective active area where electrons and holes recombine to generate DUV photons can be different from the geometrical active area, especially for devices operated by the lateral flow of carriers through ohmic contacts and an active layer in between. The effective area can be quantified by the transfer length $\left(L_{\mathrm{T}}\right)$ of carriers. ${ }^{7}$ Due to the low carrier concentration and low carrier mobility of AlGaN, the $L_{\mathrm{T}}$ is limited to only a few tens of microns at most, depending on ohmic contact resistivity (Supplementary Fig. S8). Therefore, LEDs having more mesa stripes with a smaller width, i.e., a smaller geometrical active mesa area, possess a larger effective active mesa area and hence show enhanced LOP. Thus, we anticipate that the LOP can be further enhanced by introducing narrower mesa structures for a larger sidewall area. Conditions for obtaining an optimum LOP will be discussed in the section of 'Future perspectives'.

The angle-dependent LOPs of the reference and SEE DUV LEDs are investigated to confirm the effect of incorporating the $\mathrm{Al}$ mirror on the directionality of the extracted DUV photons, as shown in Figure 6. First, the strong sidewall-emission of the DUV LED is reflected in the emission pattern of the reference DUV LED. Light emission at an angle of $30^{\circ}$ is greater than that at other angles and is consistent with the emission pattern of the $c$-plane AlN LED. ${ }^{11,12}$ In contrast, the emission pattern of the SEE DUV LED is similar to that of a conventional $c$ plane GaInN LED. Emission in the surface-normal direction, $90^{\circ}$, is significantly enhanced, while emission at angles lower than approximately $50^{\circ}$ is reduced. This is due to the reflection of DUV light by the Al mirror. Based on the SEE DUV LED chip geometry (Supplementary Fig. S9), DUV light emitted at an angle lower than approximately $45^{\circ}$ is expected to be blocked and reflected by the Al mirror. Thus, the experimentally observed enhanced (reduced) LOP at an angle higher (lower) than approximately $50^{\circ} \mathrm{can}$ be understood by the geometry of the $\mathrm{Al}$ mirror. 


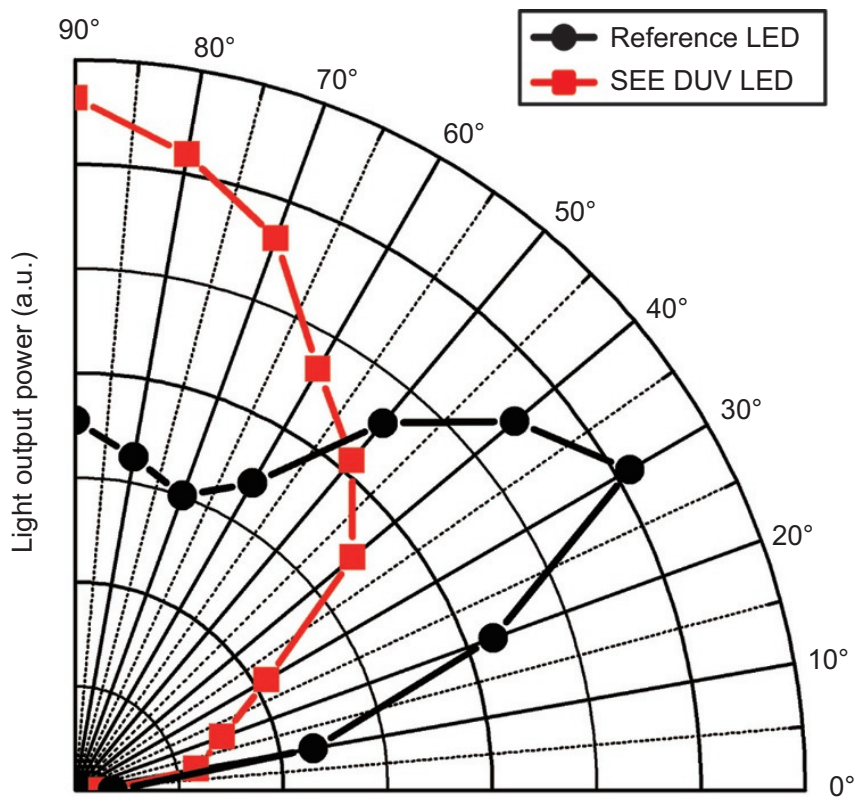

Figure 6 Angle-dependent LOP. Representative angle-dependent LOP of the reference LED and SEE DUV LED having 45 active mesa stripes. The reference LED shows strong sidewall emission with the highest LOP at an angle of $30^{\circ}$. The SEE DUV LED shows much enhanced surface-normal emission due to reflection by the AI mirrors. DUV, deep ultraviolet; LED, light-emitting diode; LOP, light output power; SEE, sidewall emission-enhanced.

Representative current-voltage $(I-V)$ characteristics of a SEE DUV LED and a reference LED with 45 stripes are shown in Figure 7a. An improved electrical property is observed in a SEE DUV LED whose operating voltage is $7.55 \mathrm{~V}$ at an injection current of $100 \mathrm{~mA}$, which is $0.25 \mathrm{~V}$ lower than that of the reference LED. Operating voltages at $100 \mathrm{~mA}$ for stripe-patterned SEE DUV LEDs and reference LEDs are summarized in Figure $7 \mathrm{~b}$ as a function of the perimeter of active mesa. For both SEE DUV LEDs and reference LEDs, the operating voltage becomes smaller with increasing perimeter, i.e., increasing $\mathrm{n}$-contact

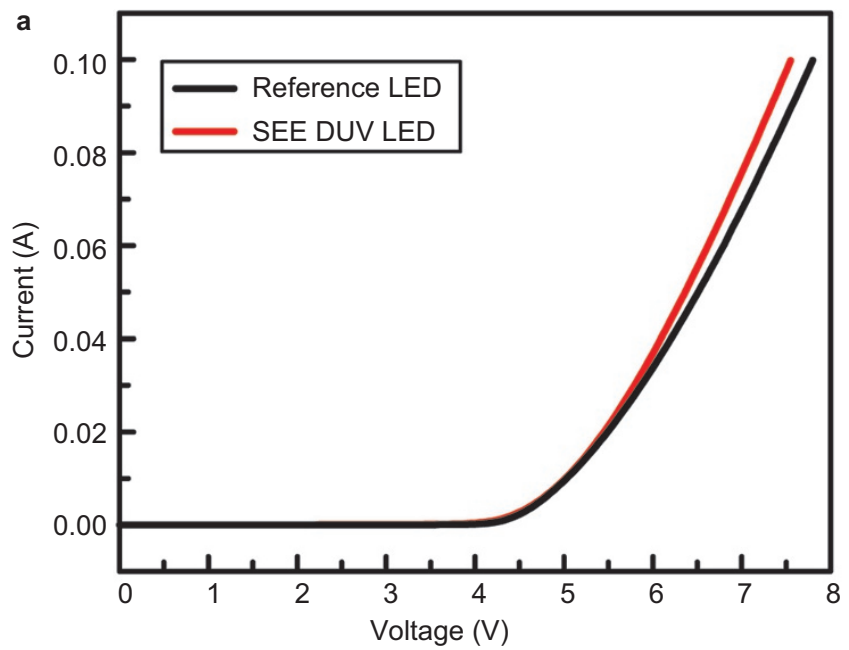

area (Table 1). In addition, SEE DUV LEDs show a lower operating voltage than the reference LED, which is attributed to two reasons: (i) better ohmic contact formation on the regrown n-type GaN than on ntype AlGaN and (ii) larger ohmic contact area in SEE DUV LEDs than in the reference LED due to the three-dimensional geometry of the regrown n-type GaN microstructures. These observations also imply that the narrower mesa stripe structure can not only increase the LEE of the SEE DUV LED but can also reduce the operating voltage by using a better $n$-contact with a larger contact area.

\section{Future perspectives}

We demonstrated a new LEE-enhancing technique for AlGaN DUV LEDs by taking advantage of their inherently strong TM-polarized sidewall emission. Based on the current study, there is much room for further enhancing the LOP and electrical properties as well as emission-pattern controllability not only for top-emitting DUV LEDs but also for bottom-emitting SEE DUV LEDs with flip-chip configuration, where the reflector geometry needs to be modified to promote emission towards the substrate.

According to Figure 5a, the amount of extracted light can be further increased by exposing a greater sidewall area of the MQW mesas by employing narrower mesa stripe structures with reduced width and a larger numbers of stripes. However, there will be an optimum width of the mesa stripes for maximum LOP without introducing a reliability issue that might be caused by the high current density when using very narrow stripes. The optimum mesa width of the stripes is anticipated to be $2 L_{\mathrm{T}}$, where $L_{\mathrm{T}}$ is the effective width of the mesa within which most carriers pass through the active region, thus determining the effective light-emitting area. ${ }^{7}$ Therefore, with a mesa stripe width of approximately $2 L_{\mathrm{T}}$, the effective active area coincides with the geometrical active area, so that the entire active mesa area can emit light uniformly without a current crowding problem and localized emission. In addition, such optimal SEE DUV LEDs with narrow stripes should reduce operating voltages as well as enhance the LEE because the $\mathrm{n}$-contact area increases as the number of stripe increases.

Meanwhile, it is experimentally confirmed that emitting DUV photons are reflected by the $\mathrm{Al}$ mirror, and the emission pattern of

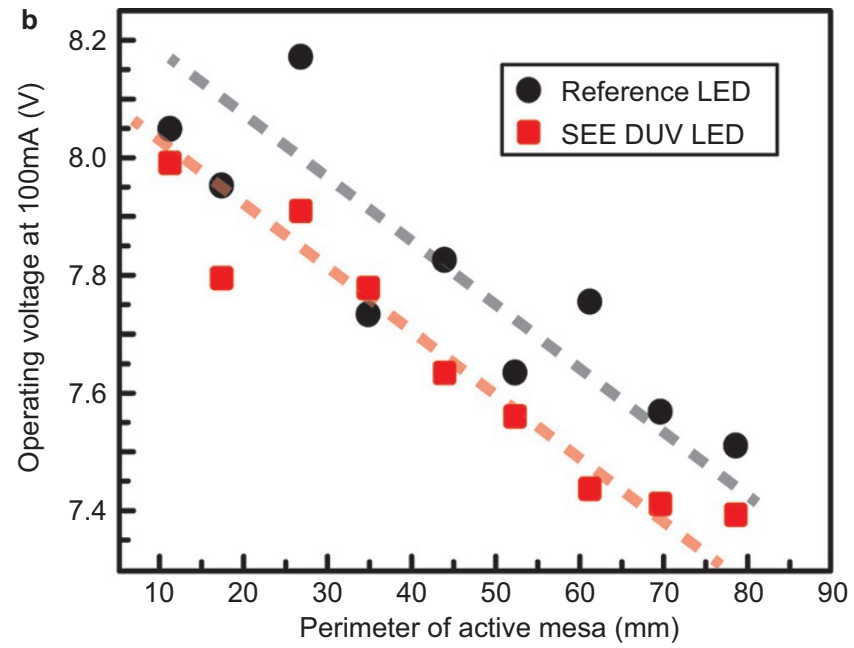

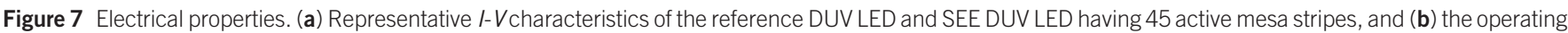

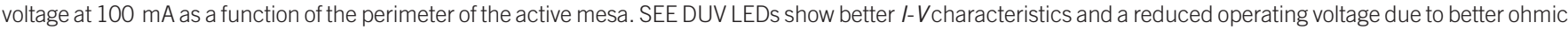

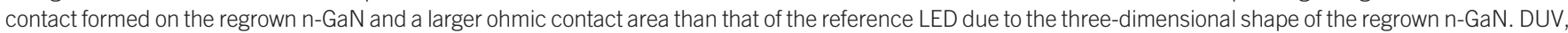
deep ultraviolet; LED, light-emitting diode; SEE, sidewall emission-enhanced. 
the SEE DUV LED is quite different from that of the reference DUV LED. Therefore, the emission pattern of the SEE DUV LED can be controlled by employing Al mirrors with various geometries. One possible way to control the shape of the $\mathrm{Al}$ mirror is by orienting the window mask along different crystal directions. Various facets can be obtained by changing the crystal direction of the window mask, and thus the emission pattern should change accordingly. For example, the $\{11 \overline{2} 2\}$ facet appears when the window mask is along the $\langle 1 \overline{1} 00\rangle$ direction in the $c$-plane. ${ }^{34}$ The incline of the surface of this facet is $54.7^{\circ}$, which is smaller than that of the $\{1 \overline{1} 01\}$ facet. Then, emission at the mid-angle range will be further improved, while the enhancement in the surface-normal direction decreases. In addition, the size of the $\mathrm{Al}$ mirror also affects the emission pattern. If a larger $\mathrm{Al}$ mirror is adopted, a higher surface-normal DUV emission can be obtained.

Top-emitting SEE DUV LEDs have the potential to exceed current DUV LEDs with a flip-chip configuration in terms of LOP. Assuming that the linear trend in Figure 5a persists, optimized top-emitting SEE DUV LEDs can be much more effective than the current flip-chip DUV LEDs. However, due to better heat dissipation in flip-chip devices, the development of bottom-emitting SEE DUV flip-chip LEDs is important for the adoption of the proposed technology. We currently develop such SEE DUV LEDs for flip-chip configuration with inclined sidewalls, reflecting photons up through the substrate by using conventional LED fabrication processes without $\mathrm{n}-\mathrm{GaN}$ regrowth; promising preliminary results are consistent with experimental data of the present work. Finally, we would like to note that the SEE DUV LED concept will be more effective when the $\mathrm{Al}$ content in the active region increases, i.e., the emission wavelength becomes shorter because of the TM-polarized light emission.

\section{CONCLUSIONS}

In summary, we propose a new LEE-enhancing strategy for AlGaNbased DUV LEDs utilizing their inherently strong sidewall emission property. The proposed SEE DUV LED includes multiple narrow active-region mesa stripes with Al-coated selective area-grown n$\mathrm{GaN}$ reflector stripes located between the mesa stripes. We experimentally observed that the LOP increases upon increasing the active-region sidewall perimeter, which is elucidated by the strong sidewall emission of the $\mathrm{AlGaN}$ active layer with high $\mathrm{Al}$ content. In addition, the Al-coated regrown $\mathrm{n}-\mathrm{GaN}$ mirror can reflect side-emitted UV photons upward so that emission along the surface-normal direction is enhanced. Furthermore, SEE DUV LEDs have a lower operating voltage than reference devices due to a less resistive n-contact on the regrown $n-G a N$, as well as a larger contact area enabled by using a three-dimensional geometry. Therefore, the optically and electrically improved AlGaN DUV LEDs are demonstrated by means of a greater LEE and lower operating voltage. Further improvements in LEE and operating voltage are expected by geometry optimization such as optimizing the distance between the mesa stripes, the width of the mesa stripes, and the size of the regrown n-GaN microstructure. We propose that the optimized mesa stripe width should be twice the carrier transfer length, $L_{\mathrm{T}}$. The same LEE-enhancing concept can be applied to DUV LEDs for flip-chip configuration so that more efficient high-power DUV LEDs can be developed.

\section{ACKNOWLEDGEMENTS}

The authors gratefully acknowledge the support by the International Collaborative R\&D Program of the Korea Institute for Advancement of Technology (KIAT) (M0000078, Development of Deep UV LED Technology for Industry and Medical Application), the Industrial Strategic Technology Development Program (10041878, Development of WPE 75\% LED device process and standard evaluation technology), and the IT R\&D Program (10035598, $180 \mathrm{~lm} \mathrm{~W} \mathrm{~W}^{-1}$ High-efficiency Nano-based LEDs) funded by the Ministry of Trade, Industry \& Energy (MOTIE, Korea)

1 Kneissl M, Kolbe T, Chua C, Kueller V, Lobo $\mathrm{N}$ et al. Advances in group III-nitridebased deep UV light-emitting diode technology. Semicond Sci Technol 2011; 26: 014036.

2 Khan MA. AIGaN multiple quantum well based deep UV LEDs and their applications. Phys Status Solidi A 2006; 203: 1764-1770.

3 Hirayama H, Fujikawa S, Noguchi N, Norimatsu J, Takano T et al. 222-282nm AIGaN and InAIGaN-based deep-UV LEDs fabricated on high-quality AIN on sapphire. Phys Stat Sol A 2009; 206: 1176-1182.

4 Decker C. The use of UV irradiation in polymerization. Polym Int 1998; 45: 133-141.

5 Lin BJ. Deep UV lithography. J Vac Sci Technol 1975; 12: 1317-1320.

6 Takeda M, Furuki M, Yamatsu H, Kashiwagi T, Aki Y et al. Deep UV mastering using an all-solid-state 266nm laser for an over 20GBytes/layer capacity disk. Jpn J App/ Phys 1999; 38: 1837-1838.

7 Schubert EF. Light-Emitting Diodes. New York: Cambridge Univ. Press; 2006.

8 Khan MA, Shatalov M, Maruska HP, Wang HM, Kuokstis E. III-Nitride UV devices. Jpn J Appl Phys 2005; 44: 7191-7206.

9 Shur MS, Gaska R. Deep-ultraviolet light-emitting diodes. IEEE Trans Electron Devices 2010; 57: 12-25.

10 Shatalov M, Sun W, Lunev A, Hu X, Dobrinsky A et al. AIGaN deep-ultraviolet lightemitting diodes with external quantum efficiency above 10\%. Appl Phys Exp 2012; 5: 082101.

11 Taniyasu Y, Kasu M. Improved emission efficiency of 210-nm deep-ultraviolet aluminum nitride light-emitting diode. NTT Tech Rev 2010; 8: 1-5.

12 Taniyasu Y, Kasu M. Surface $210 \mathrm{~nm}$ light emission from an AIN p-n junction lightemitting diode enhanced by A-plane growth orientation. App/ Phys Lett 2010; 96: 221110.

13 Nam KB, Li J, Nakarmi ML, Lin JY, Jiang HX. Unique optical properties of AIGaN alloys and related ultraviolet emitters. Appl Phys Lett 2004; 84: 5264.

14 Shatalov M, Lunev A, Hu X, Bilenko O, Gaska I et al. Performance and applications of deep UV LED. Int J High Speed Electron Syst 2012; 21: 1250011.

15 Maeda N, Hirayama H. Realization of high-efficiency deep-UV LEDs using transparent p-AIGaN contact layer. Phys Status Solidi C 2013; 10: 1521-1524.

16 Chen J, Grandusky JR, Mendrick MC, Gibb S, Kim YS et al. Enhanced photon extraction efficiency in $260 \mathrm{~nm}$ pseudomorphic AIN-based ultraviolet light emitting diodes. In: Proceedings of International Semiconductor Device Research Symposium 2011 (ISDRS 2011); 7-9 December 2011; College Park, MD, USA. IEEE: New York, USA, 2011; 1-2. DOI: 10.1109/ISDRS.2011.6135384.

17 Kim BJ, Jung H, Shin J, Mastro MA, Eddy CR Jr et al. Enhancement of light extraction efficiency of ultraviolet light emitting diodes by patterning of $\mathrm{SiO}_{2}$ nanosphere arrays. Thin Solid Films 2009; 517: 2742-2744.

18 Dong P, Yan J, Wang J, Zhang Y, Geng C et al. 282-nm AIGaN-based deep ultraviolet light-emitting diodes with improved performance on nano-patterned sapphire substrates. App/ Phys Lett 2013; 102: 241113.

19 Yan X, Shatalov M, Saxena T, Shur MS. Deep-ultraviolet tailored- and low-refractive index antireflection coatings for light-extraction enhancement of light emitting diodes. J Appl Phys 2013; 113: 163105.

20 Shakya J, Kim KH, Lin JY, Jiang HX, Enhanced light extraction in III-nitride ultraviolet photonic crystal light-emitting diodes. App/ Phys Lett 2004; 85: 142

21 Gao N, Huang K, Li J, Li S, Yang X, et al. Surface-plasmon-enhanced deep-UV light emitting diodes based on AIGaN multi-quantum wells. Sci Rep 2012; 2: 816.

22 Ryu HY, Choi IG, Choi HS, Shim JI. investigation of light extraction efficiency in AIGaN deep-ultraviolet light-emitting diodes. App/ Phys Expr 2013; 6: 062101.

23 Zheleva TS, Nam OH, Bremser MD, Davis RF. Dislocation density reduction via lateral epitaxy in selectively grown GaN structures. App/ Phys Lett 1997; 71: 2472.

24 Kapolnek D, Keller S, Vetury R, Underwood RD, Kozodoy P et al. Anisotropic epitaxial lateral growth in GaN selective area epitaxy. Appl Phys Lett 1997; 71: 1204.

25 Kato Y, Kitamura S, Hiramatsu K, Sawaki N. Selective growth of wurtzite GaN and $\mathrm{Al}_{x} \mathrm{Ga}_{1-x} \mathrm{~N}$ on $\mathrm{GaN} /$ sapphire substrates by metalorganic vapor phase epitaxy. J Cryst Growth 1994; 144: 133-140.

26 Coldren LA, Corzine SW. Diode Lasers and Photonic Integrated Circuits. New York: Wiley-VCH; 1995.

27 Braunstein R, Kane EO. The valence band structure of the III-V compounds. J Phys Chem Solids 1962; 23: 1423-1431.

28 Chuang SL, Chang CS. k.p method for strained wurtzite semiconductors. Phys Rev $B$ 1996; 54: 2491-2504.

29 Northrup JE, Chua CL, Yang Z, Wunderer T, Kneissl M et al. Effect of strain and barrier composition on the polarization of light emission from AIGaN/AIN quantum wells. App/ Phys Lett 2012; 100: 021101.

30 Mathews PM, Venkatesan K. A Textbook of Quantum Mechanics. New Delhi: McGrawHill; 1976.

31 Guo X, Schubert EF. Current crowding and optical saturation effects in GalnN/GaN light-emitting diodes grown on insulating substrates. App/ Phys Lett2001; 78: 33373339.

32 Guo X, Schubert EF. Current crowding in GaN/InGaN light emitting diodes on insulating substrates. J App/ Phys 2001; 90: 4191-4195. 
33 Adivarahan V, Wu S, Sun WH, Mandavilli V, Shatalov MS et al. High-power deep ultraviolet light-emitting diodes based on a micro-pixel design. App/ Phys Lett 2004; 85: 1838-1840.

34 Miyake $\mathrm{H}$, Motogaito A, Hiramatsu K. Effects of reactor pressure on epitaxial lateral overgrowth of GaN via low-pressure metalorganic vapor phase epitaxy. Jpn J App/ Phys 1999; 38: L1000-L1002. (c) (i) (2) This work is licensed under a Creative Commons Attribution-

BY NG SA NonCommercial-ShareAlike 3.0 Unported License. The images or other third party material in this article are included in the article's Creative Commons license, unless indicated otherwise in the credit line; if the material is not included under the Creative

Commons license, users will need to obtain permissing from the license holder to reproduce the material. To view a copy of this license, visit http://creativecommons.org/licenses/by-nc-sa/3.0/

Supplementary information for this article can be found on the Light: Science \& Applications' website (http://www.nature.com/lsa/). 